\title{
Reflujo hepatoyugular
}

\section{Hepatojugular reflux}

\author{
Alfredo Pinzón-Junca • Bogotá, D.C. (Colombia)
}

DOI: https://doi.org/10.36104/amc.2019.0000

En ocasiones se genera confusión al utilizar términos con similitud fonética. Este parecer ser el caso de las palabras reflujo y reflejo, que lleva a algunos al uso de la expresión inadecuada: reflejo hepatoyugular.

Reflujo: (Del latín medieval refluxum [re 'hacia atrás' 'con intensidad' + fluc 'fluir' + -sum]; documentado en español desde 1424 en la forma refluxo) [inglés: reflux]:

1) s. m. Med. Flujo retrógrado, y habitualmente anómalo, de un líquido orgánico en el interior de un conducto o una víscera hueca.

2) Movimiento de descenso de la marea.

Reflejo: (Del latín reflexus 'acción de volver hacia atrás', 'retroceso' 'doblado hacia atrás' [re 'repetición' + flec(tere) 'doblar' 'curvar' + -sum]; usado en español desde 1508) [inglés: reflex]):

1) s.m. Respuesta involuntaria, simple o compleja, a cualquier estímulo sensitivo, sensorial o psíquico.

2) adj. Dicho de un movimiento corporal, una secreción, un sentimiento, etc.: Que se produce involuntaria o automáticamente como respuesta a un estímulo.

Según esto, la expresión correcta es reflujo hepatoyugular. Se refiere a la elevación de la presión venosa yugular cuando se presiona el hipocondrio derecho, por un tiempo que varía de 10 a 30 segundos, y se manifiesta por ingurgitación de las venas yugulares de más de 3-4 cm, que cae cuando se suspende la maniobra. Esta prueba fue descrita por W. Pasteur en 1885 como un signo de la insuficiencia tricúspidea, aunque en 1898 Rondot descubrió que pacientes con válvula triscúspide normal podían presentarlo, y en 1925 otros clínicos notaron que la presión sobre cualquier área del abdomen, no solamente la hepática, podía desencadenarlo. Actualmente esta maniobra es conocida como prueba hepatoyugular, o mejor aún abdominoyugular, ya que no hay un verdadero reflujo involucrado. En pacientes con disnea, es útil para predecir falla cardiaca izquierda (LR+ 6.0; LR- 0.78) y sugiere presión pulmonar capilar en cuña mayor a $15 \mathrm{mmHg}$ (LR+ 6.7; LR- 0.08) [Sensibilidad: 55-84\%, especificidad: 83-98\%]. Cabe aclarar que no es específica de ninguna enfermedad, aunque las causas comunes de un hallazgo positivo incluyen pericarditis constrictiva, infarto ventricular derecho y cardiomiopatía restrictiva.

\section{Fuentes:}

- McGee S. Evidence-based physical diagnosis. Elsevier Saunders. 2012; 301

- Wiese J. The abdominojugular reflux sign. Am J Med 2000; 109:59-61

- Pasteur W. Note on a new physical sign of tricuspid regurgitation. Lancet 1885; 2:524

- Real Academia Nacional de Medicina. Diccionario de Términos Médicos. Editorial Médica Panamericana. 2012

- Diccionario de la Real Academia Española de la Lengua, disponible en: www.rae.es

\footnotetext{
* Envíe sus inquietudes, sugerencias o comentarios a: contacto@actamedicacolombiana.com - alfpin@hotmail.com Dr. Alfredo Pinzón-Junca: Especialista en Medicina Interna y Psicoanálisis. Hospital Universitario de La Samaritana y Hospital Simón Bolívar. Coordinador del Consejo de Acreditación y Recertificación de la ACMI ${ }^{\circledR}$. Bogotá, D.C. (Colombia). E-mail: alfpin@hotmail.com

Recibido: 17/IX/2019 Aceptado: 19/IX/2019
} 